\title{
Three-dimensional shock spectrum of critical component for nonlinear packaging system
}

\author{
Jun Wang ${ }^{\mathrm{a}, \mathrm{b}}$, Zhi-Wei Wang ${ }^{\mathrm{c}, *}, \mathrm{Li}-\mathrm{Xin} \mathrm{Lu}^{\mathrm{a}, \mathrm{b}}$, Yong Zhu ${ }^{\mathrm{c}}$ and Yong-Guang Wang ${ }^{\mathrm{a}}$ \\ ${ }^{a}$ Department of Packaging Engineering, Jiangnan University, Wuxi, P.R. China \\ ${ }^{\mathrm{b}}$ Key laboratory of food packaging techniques \& safety of China national packaging corporation, Wuxi, P.R. China \\ ${ }^{\mathrm{c}}$ Packaging Engineering Institute, Jinan University, Zhuhai, P.R. China
}

Received 22 July 2008

Revised 2009

\begin{abstract}
To evaluate the shock characteristics of critical component for a nonlinear packaging system, a new concept of threedimensional shock spectrum was proposed. Three key coordinate parameters, such as the nondimensional pulse duration, the frequency parameter ratio and the ratio of the maximum response acceleration to the peak pulse acceleration, were governed in a novel dynamical mathematical model. It is shown that the shock response of critical component is weakened owning to the decrease in the defined system parameter. Furthermore, at low frequency parameter ratio, the enhancement of the damping ratio of the critical component leads to the decrease in the peak of the shock response, which can also be reduced by the increase in damping ratio of cushioning pad at both lower and higher frequency parameter ratios. The discussion and analysis provide some insights into the design of cushioning packaging as well.
\end{abstract}

Keywords: Critical component, Two-degree-of-freedom, Nonlinear, Influencing factors, Three-dimensional shock spectrum

\section{Introduction}

The two-dimensional damage boundary concept (DBC) proposed by Newton [1] is the foundation for the present packaging design. Despite its popularity, some basic assumptions of this theory has been questioned: (1) the product packaging system was considered to be linear, undamped spring-mass single-degree-of-freedom system; (2) there were no restrictions to the deformation of cushioning pad. These may not be valid because of the complexity of products configuration and the diversity of cushioning materials. Several researchers developed some modified versions of the concept. Burgess [2] presented a fatigue damage boundary (FDB) method to describe the effect of multiple shocks on damage of products. Goodwin [3] tested the fatigue damage boundary of a camera. In recent years, the concept of displacement damage boundary was applied by Wang [4] to study the deformation of cushioning materials. And a further extension was made by Wang [5] to evaluate the dropping damage of products for both linear and nonlinear packaging systems. Most recently, Lu [6] established a concept of bruising fragility and bruising boundary for fruits, which can be widely applied to other similar viscoelastic products.

Since the damage boundary is a very conservative approach to evaluate the damage potential of shock to product, the concept of shock response spectrum [7] (SRS) was introduced into packaging design from other engineering disciplines. SRS can predict the response of packaged product with a known natural frequency, which didn't focus on the input shock impulse but the response of product, this is a departure from the traditional method. In recent years, packaging researchers have become increasingly interested in shock response spectrum for packaged product.

\footnotetext{
*Corresponding author: Zhi-Wei Wang, Packaging Engineering Institute, Jinan University, Zhuhai 519070, P.R. China. E-mail: packei@ yahoo. com.cn.
} 


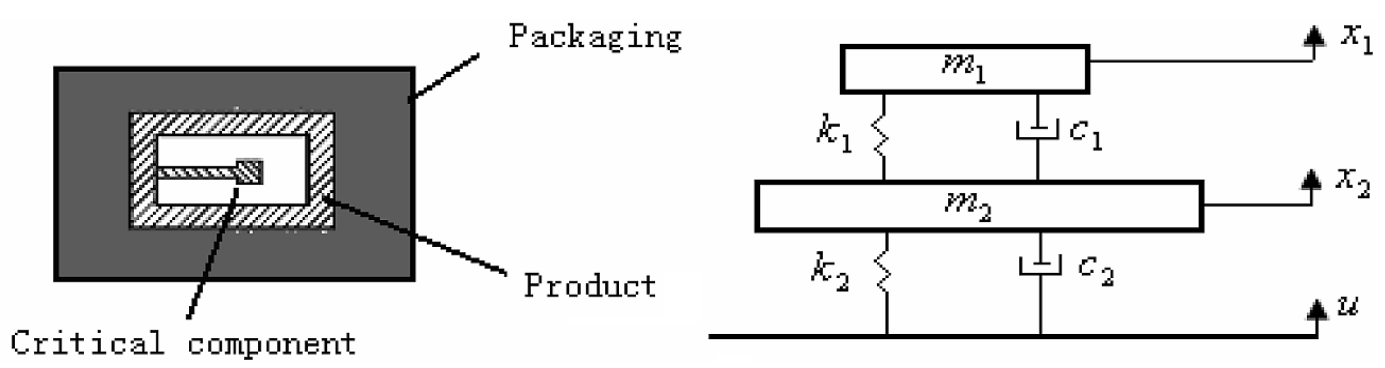

Fig. 1. Model of packaging system with critical component.

Wang [8] suggested a general approach to obtain the SRS and DBC for a typical nonlinear packaging system and discussed the influencing factors of shock response of the packaged product.Daum [9] incorporated SRS with a fatigue model and extended the SRS concept to evaluate the damage potential of repetitive shocks to ductile products.

The most serious limitation in the previous research is the assumption that the packaged product can be modeled as a single mass block. However, most products, especially those mechanical and electronic products, are composed of large numbers of elements, and the damage generally occurs at the so-called critical component. The previous research failed to evaluate the damage potential of shock to packaged product with critical component. In this paper, the critical component, the main body of product and the cushioning packaging are modeled as a whole system. For a critical component, the shock characteristics of a nonlinear packaging system with most actual nonlinear cushioning materials are investigated not been discussed preciously. The objective of this paper is to develop a damage evaluation approach for typical nonlinear packaging system with critical component.

\section{Modeling and equations}

The model of packaging system with critical component is depicted in Fig. 1, in which the packaging is idealized as a nonlinear spring with stiffness coefficient $k_{2}$ and damping ratio $c_{2}$. The joint part between critical component $\left(m_{1}\right)$ and main body of product $\left(m_{2}\right)$ is idealized as a linear spring with stiffness coefficient $k_{1}$ and damping ratio $c_{1}$. And $x_{1}, x_{2}, u$ represent respectively the displacement of the critical component, the main body and the foundation.

The motion of the system is governed by

$$
\begin{aligned}
& m_{1} \ddot{x}_{1}=k_{1}\left(x_{2}-x_{1}\right)+c_{1}\left(\dot{x}_{2}-\dot{x}_{1}\right) \\
& m_{2} \ddot{x}_{2}=f\left(u-x_{2}\right)+c_{2}\left(\dot{u}-\dot{x}_{2}\right)-k_{1}\left(x_{2}-x_{1}\right)-c_{1}\left(\dot{x}_{2}-\dot{x}_{1}\right)
\end{aligned}
$$

where the restoring force $f\left(u-x_{2}\right)$ are expressed in Table 1 for typical nonlinear packaging system. The base excitation $u^{\prime \prime}(t)$ is assumed to be a half-sine pulse described as

$$
u^{\prime \prime}=u_{0 m}^{\prime \prime} \sin { }_{t_{0}}^{\pi t} \Delta \mathrm{H}\left(t, t_{0}\right)
$$

$u_{0 m}^{\prime \prime}$ and $t_{0}$ denote the magnitude and duration of the excitation pulse, respectively.

To simplify these equations, we introduce the new nondimensional variables

$$
\begin{aligned}
& \delta_{1}=\left(x_{2}-x_{1}\right) / L \\
& \delta_{2}=\left(u-x_{2}\right) / L \\
& \tau=t / T
\end{aligned}
$$

where $T=\sqrt{ } m_{2} / k_{2}, L$ is chosen according to the type of cushioning materials, which is given in Table 1 .

Furthermore, frequency parameter of the critical component and product are respectively defined as

$$
\omega_{1}=\sqrt{ } k_{1} / m_{1}
$$


Table 1

The restoring force, system parameters of different types of cushioning materials

$\begin{array}{cccc}\text { Type } & \text { Cubic materials } & \text { Tangent materials } & \text { Hyperbolic tangent materials } \\ f(x) & k_{2} x+r x^{3} & 2 k_{2} d_{b} \tan { }_{2 d_{b}}^{\pi} x & F_{0} \text { th }{ }_{F_{0}} x \\ L & \sqrt{ } k_{2} / r & 2 d_{b} / \pi & F_{0} / k_{2} \\ \beta & m_{2} r^{1 / 2 k_{2}^{-3} / 2} & \pi m_{2} / 2 k_{2} d_{b} & m_{2} / F_{0} \\ F\left(\delta_{2}\right) & \delta_{2}+\delta_{2}^{3} & \tan \delta_{2} & \operatorname{th} \delta_{2}\end{array}$

$\omega_{2}=\sqrt{ } k_{2} / m_{2}$

Then, frequency parameter ratio and mass ratio are separately defined as

$$
\begin{aligned}
& \lambda_{1}=\omega_{1} / \omega_{2} \\
& \lambda_{2}=m_{1} / m_{2}
\end{aligned}
$$

And damping ratio of the critical component and cushioning pad are respectively defined as

$$
\begin{aligned}
\zeta_{1} & =\begin{array}{c}
c_{1} \\
2 \sqrt{ } m_{1} k_{1}
\end{array} \\
\zeta_{2} & =\begin{array}{c}
c_{2} \\
2 \sqrt{ } m_{2} k_{2}
\end{array}
\end{aligned}
$$

Substituting all of the parameters defined above into Eq. (1), yields the nondimensional form of the motion equations:

$$
\begin{aligned}
& \ddot{\delta}_{1}=F\left(\delta_{2}\right)+2 \zeta_{2} \dot{\delta}_{2}-\left(\lambda_{2}+1\right) \lambda_{1}^{2} \delta_{1}-2\left(\lambda_{2}+1\right) \lambda_{1} \zeta_{1} \dot{\delta}_{1} \\
& \ddot{\delta}_{2}=\ddot{U}+\lambda_{2} \lambda_{1}^{2} \delta_{1}+2 \lambda_{1} \lambda_{2} \zeta_{1} \dot{\delta}_{1}-F\left(\delta_{2}\right)-2 \zeta_{2} \dot{\delta}_{2}
\end{aligned}
$$

where $F\left(\delta_{2}\right)$ is the nondimensional form of the restoring forces of cushioning materials, as shown in Table 1 . $\ddot{U}$ denotes the nondimensional base excitation acceleration, which can be expressed as:

$$
\ddot{U}=\beta u_{0 m}^{\prime \prime} \sin \left(\pi \tau / \tau_{0}\right) \Delta H\left(\tau, \tau_{0}\right)
$$

where $\beta=T^{2} / L$ is defined as the system parameter.

\section{Numerical results and discussion}

The dynamics of the two-mass system with two degrees of freedom has been widely discussed for a long time. Both analytical solution and approximate analytical solutions to the dynamical equations have been developed [1015]. Here, the numerical solution to Eq. (7) is obtained by applying the Runge-Kutta method. Based on the results, the effect of frequency parameter ratio on shock response acceleration-time history of both critical component and main body is studied.

The strong dependence nature of acceleration-time response history on frequency parameter ratio is found, as shown in Fig. 2, which may account for Fig. 3(a). Figure 3(a) indicates that the maximum response acceleration $\left(\alpha_{1}\right)$ of the critical component increases then decreases with the increase of frequency parameter ratio. It's also noted that the maximum response acceleration of critical component in Fig. 2(b) is larger than those of Fig. 2(a) and Fig. 2(c). This sensitivity of the response to the frequency parameter ratio has to be taken into account when designing cushioning packaging for protection of critical component.

The influence of mass ratio on shock response of the critical component may be significantly affected by frequency parameter ratio. As can be read from Fig. 3(b), the maximum response acceleration of critical component decreases from 1.5 to 0.5 with the increase of mass ratio from 0.02 to 0.2 at a frequency parameter ratio of 1 , while little effect can be observed at other values of frequency parameter ratio. 


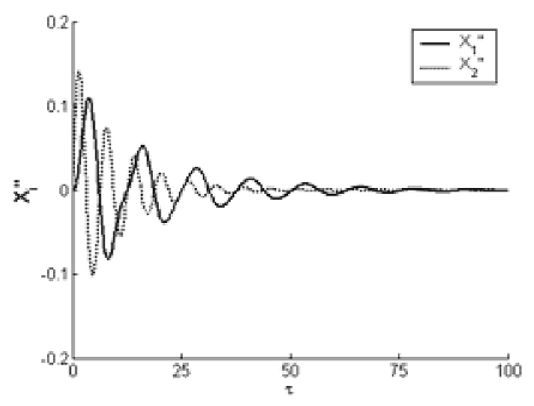

(a) $\lambda_{1}=0.5$

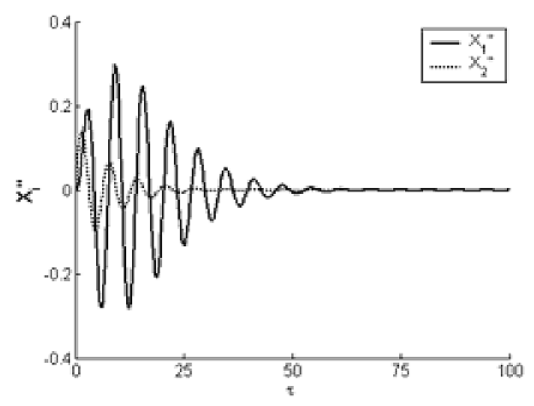

(b) $\lambda_{1}=1$

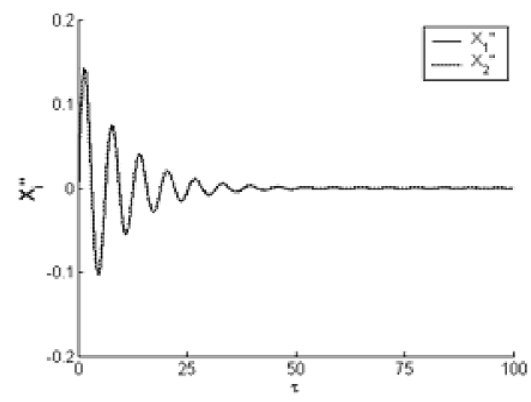

(c) $\lambda_{1}=10$

Fig. 2. Acceleration-time response history of critical component and main body at different frequency parameter ratios. Here, $\beta \ddot{\psi}_{m}^{\prime \prime}=0.5$, $\tau_{0}=0.5, \lambda_{2}=0.01, \zeta_{1}=0.1, \zeta_{2}=0.1$.

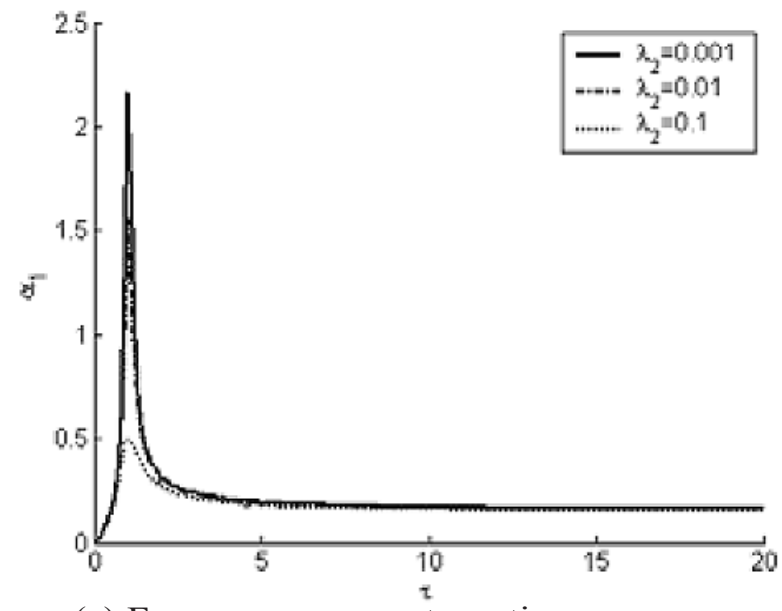

(a) Frequency parameter ratio

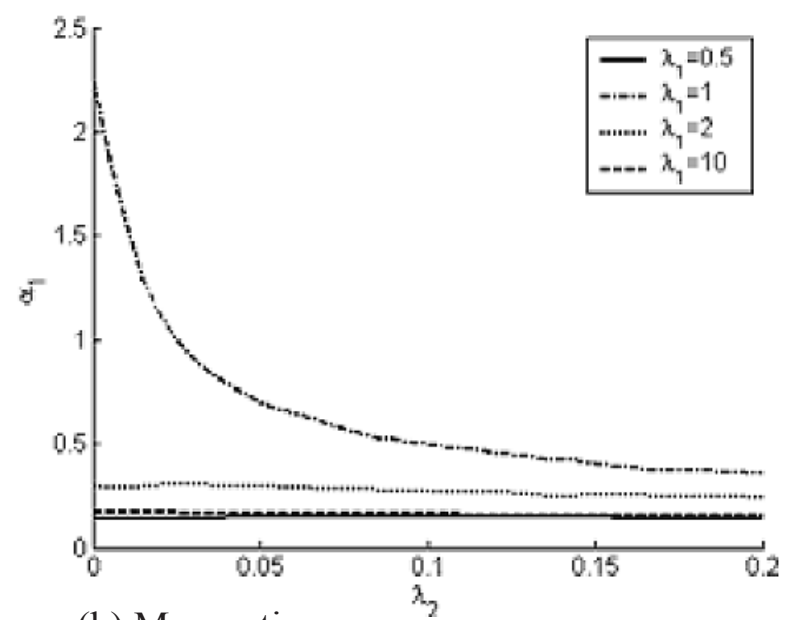

(b) Mass ratio

Fig. 3. Maximum acceleration of critical component $\left(\alpha_{1}\right)$ as a function of frequency parameter ratio $\left(\lambda_{1}\right)$ and mass ratio $\left(\lambda_{2}\right)$. Here, $\beta u_{0 m}^{\prime \prime}=0.5$, $\tau_{0}=0.5, \zeta_{1}=0, \zeta_{2}=0$.

The above results can be better explained by referring to the so-called shock response spectra. Those are plots for the peak response of a spring-mass-damper system to shock load as a function of the natural period of the system. These plots are available for various shock shapes and damping ratios. Figure 4 shows an example SRS for critical component. The figure shows the shock amplification factor $\alpha_{1} / u_{0 m}^{\prime \prime}$ (denoted as $\gamma_{1}$ ), which is the ratio of maximum response acceleration of critical component induced in the responding packaging system to peak acceleration of the excitation pulse, versus nondimensional pulse duration $\omega_{2} t_{0}$ (denoted as $\tau_{0}$ ). We note from this figure that if $\tau_{0}$ is large, then $\gamma_{1}$ is close to unity. This corresponds to quasi-static loading case. On the other hand, if $\tau_{0}$ is small, $\gamma_{1}$ is different from unity, corresponding to dynamical loading cases. Another interesting phenomenon observed is that the shock response spectrum of critical component exhibits difference at different values of frequency parameter ratio. The peak of SRS is found to be evidently affected by frequency parameter ratio.

To evaluate the damage potential of shock to critical component more effectively, we suggest a three-dimensional shock spectrum concept, which incorporates not only the nondimensional pulse duration $\left(\tau_{0}\right)$ and the ratio of the maximum response acceleration of the critical component to the peak pulse acceleration $\left(\gamma_{1}\right)$, but also frequency parameter ratio $\left(\lambda_{1}\right)$. Figure 5 depicts the three-dimensional shock spectrum of critical component at different nondimensional pulse peaks.

From Fig. 5, we can see that the peak of the shock response of critical component at each frequency parameter ratio increases along with the increase of $\beta u_{0 m}^{\prime \prime}$, which implies that the shock response of critical component can be 


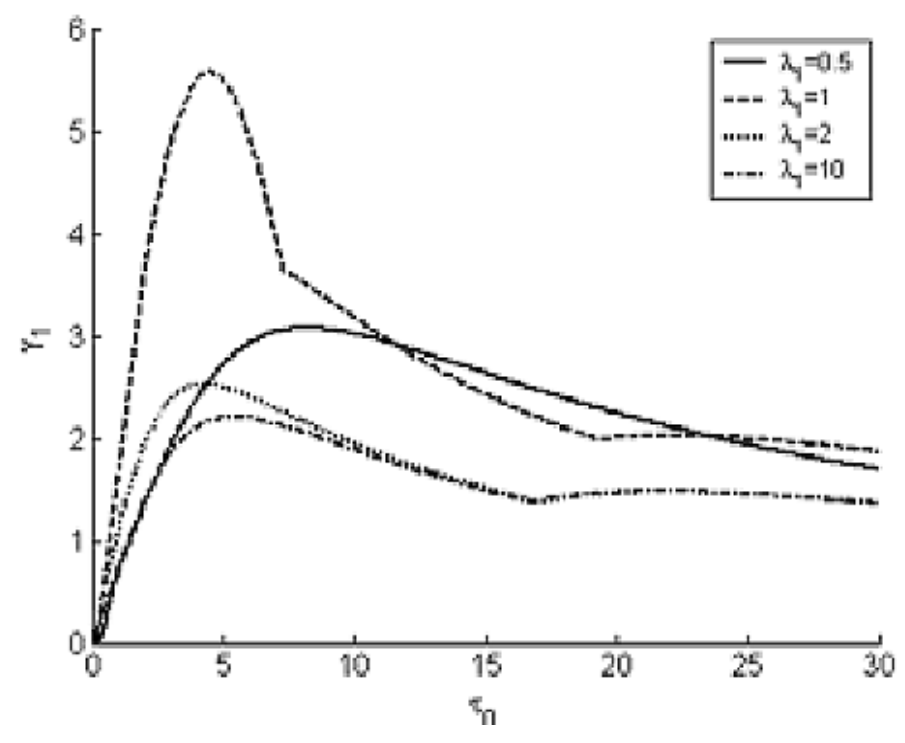

Fig. 4. SRS of Critical component at different frequency parameter ratio $\left(\lambda_{1}\right)$. Here, $\beta u_{0 m}^{\prime \prime}=0.5, \lambda_{2}=0.1, \zeta_{1}=0, \zeta_{2}=0$.

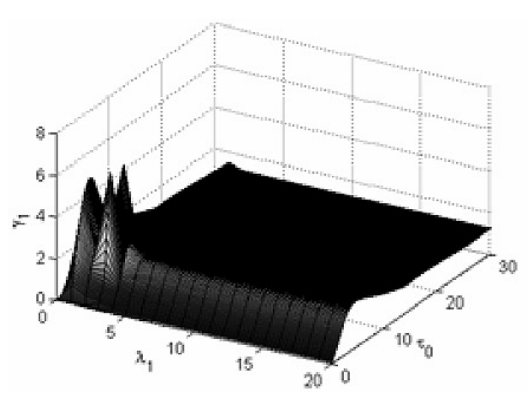

(a) $\beta u_{0 m}^{\prime \prime}=0.5$

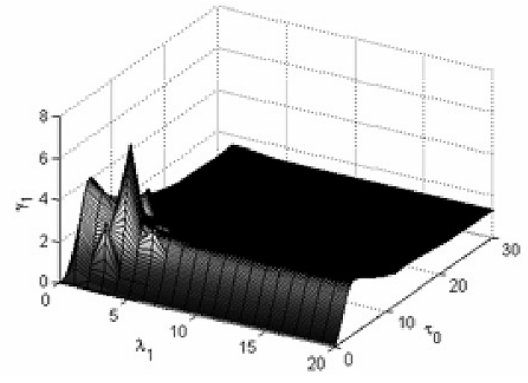

(b) $\beta u_{0 m}^{\prime \prime}=1$

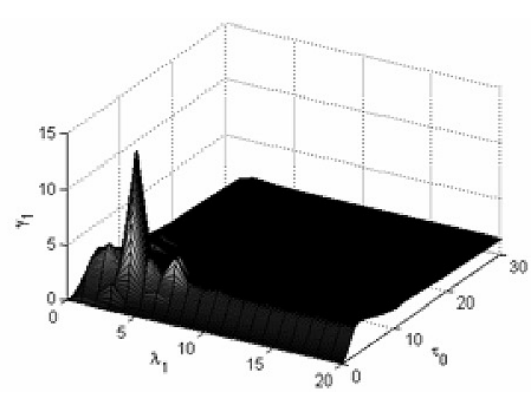

(c) $\beta u_{0 m}=1.5$

Fig. 5. Three-dimensional shock spectrum of critical component at different values of pulse peak $\left(\beta \ddot{0}_{m}^{\prime \prime}\right)$. Here, $\lambda_{2}=0.1, \zeta_{1}=0, \zeta_{2}=0$.

effectively reduced by decreasing the system parameter $\beta$. This similar conclusions can be drawn for other nonlinear packaging systems.

Next, we continue to discuss the effect of damping ratio of critical component and cushioning pad on shock response of critical component.

As depicted in Fig. 6, increasing damping ratio of critical component $\left(\zeta_{1}\right)$ leads to reduction of shock response at low frequency parameter ratio $\left(\lambda_{1}<4\right.$ in Fig. 6), while little effect can be observed at higher frequency parameter ratio.

Figure 7 shows the three-dimensional shock spectrum of the critical component at different values of the damping ratio of cushioning pad $\left(\zeta_{2}\right)$. It's found that the peak of 3D-SRS of critical component decreases with the increase of $\zeta_{2}$. It is concluded here that the damping ratio of cushioning pad can play a crucial rule in the protection of critical component. Packaging designer can effectively protect the critical component from being damaged by selecting cushioning materials with high damping.

The three-dimensional shock spectrum concept proposed above seems to be suitable for characterizing the shock response of critical component. However, it remains of no practical use unless we can obtain the values of joint parameters between the critical component and the main body, which are the spring constant $k_{1}$ and the damping coefficient $c_{1}$ in the present case. Now, we continue to discuss this problem. 


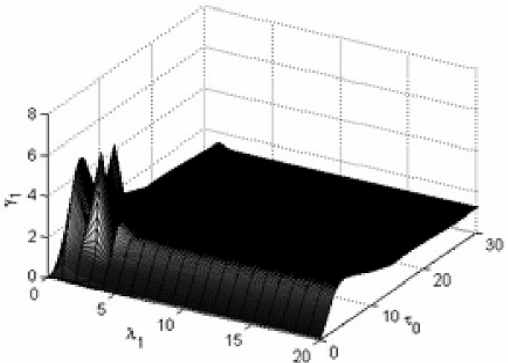

(a) $\zeta_{1}=0$

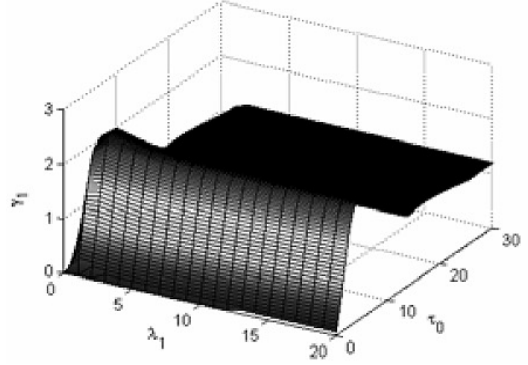

(d) $\zeta_{1}=0.5$

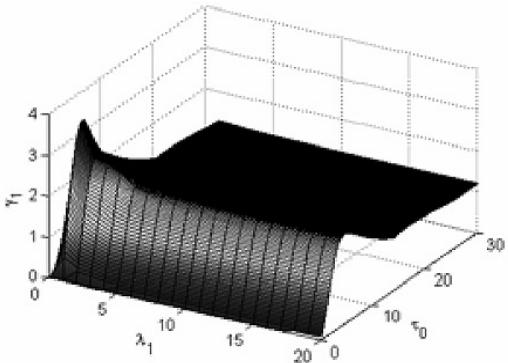

(b) $\zeta_{1}=0.1$

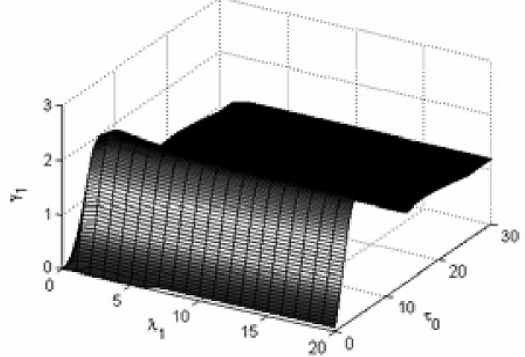

(e) $\zeta_{1}=0.8$

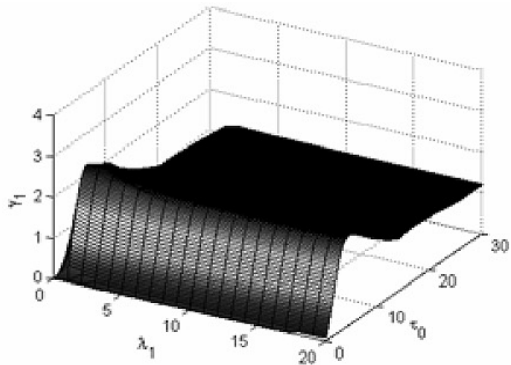

(c) $\zeta_{1}=0.3$

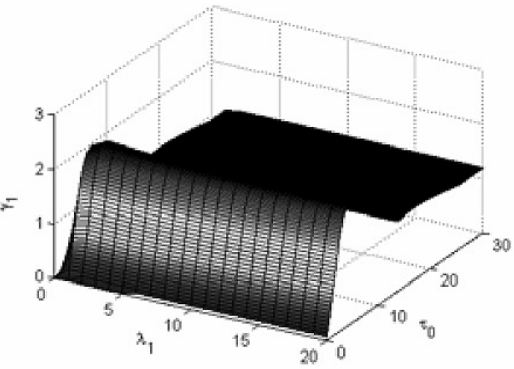

(f) $\zeta_{1}=1$

Fig. 6. Effect of damping ratio of critical component $\left(\zeta_{1}\right)$ on three-dimensional shock spectrum of critical component. Here, $\lambda_{2}=0.1, \zeta_{2}=0$.

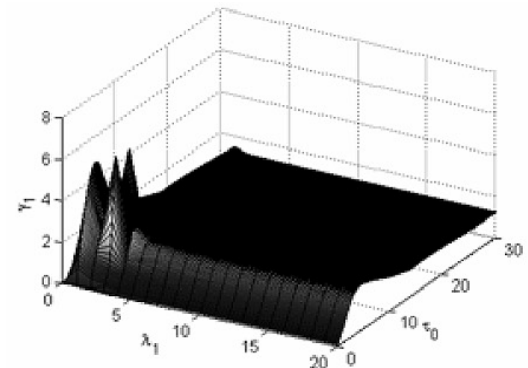

(a) $\zeta_{2}=0$

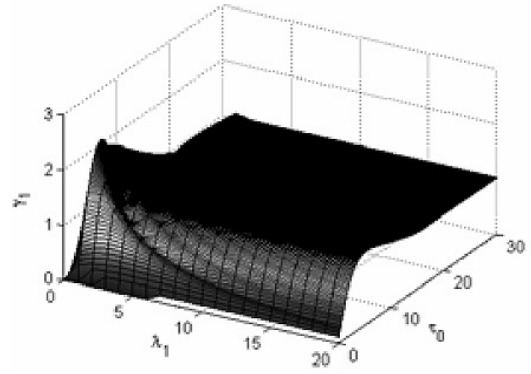

(d) $\zeta_{2}=0.5$

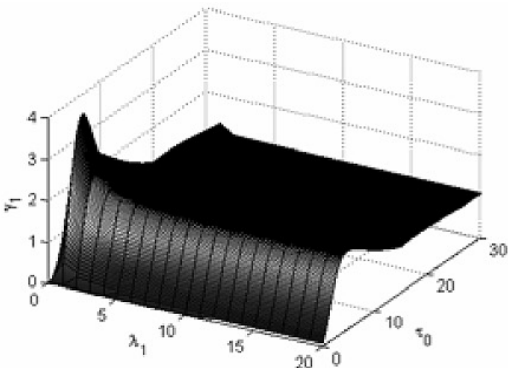

(b) $\zeta_{2}=0.1$

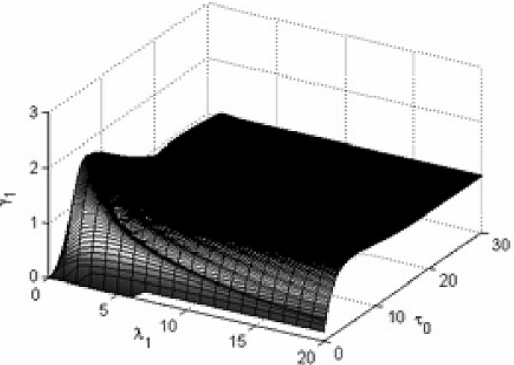

(e) $\zeta_{2}=0.8$

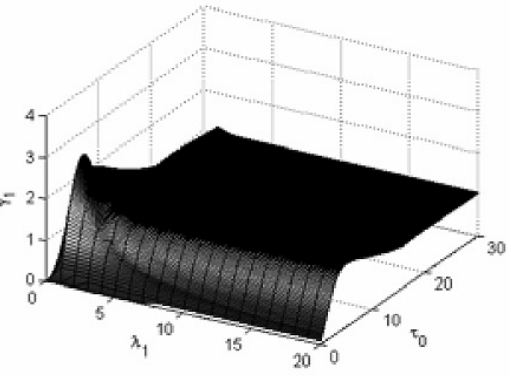

(c) $\zeta_{2}=0.3$

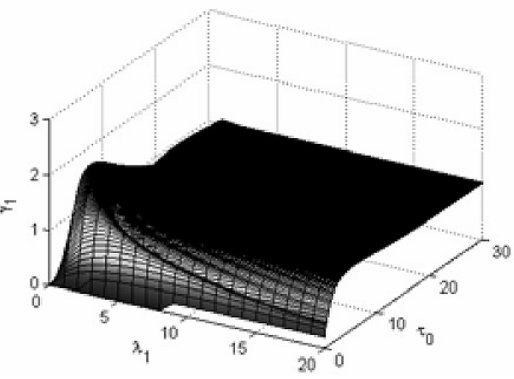

(f) $\zeta_{2}=1$

Fig. 7. Effect of damping ratio of cushioning pad $\left(\zeta_{2}\right)$ on three-dimensional shock spectrum of critical component. Here, $\beta u_{0 m}^{\prime \prime}=0.5, \lambda_{2}=0.1$, $\zeta_{1}=0$. 


\section{D}

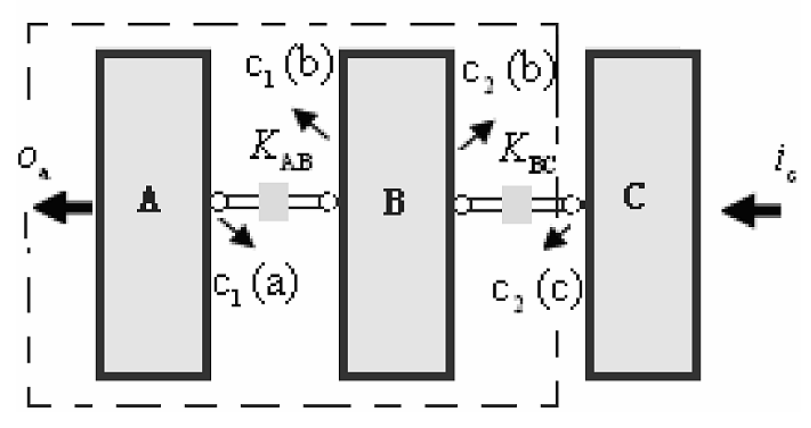

Fig. 8. One-coordinate coupled three-substructure system.

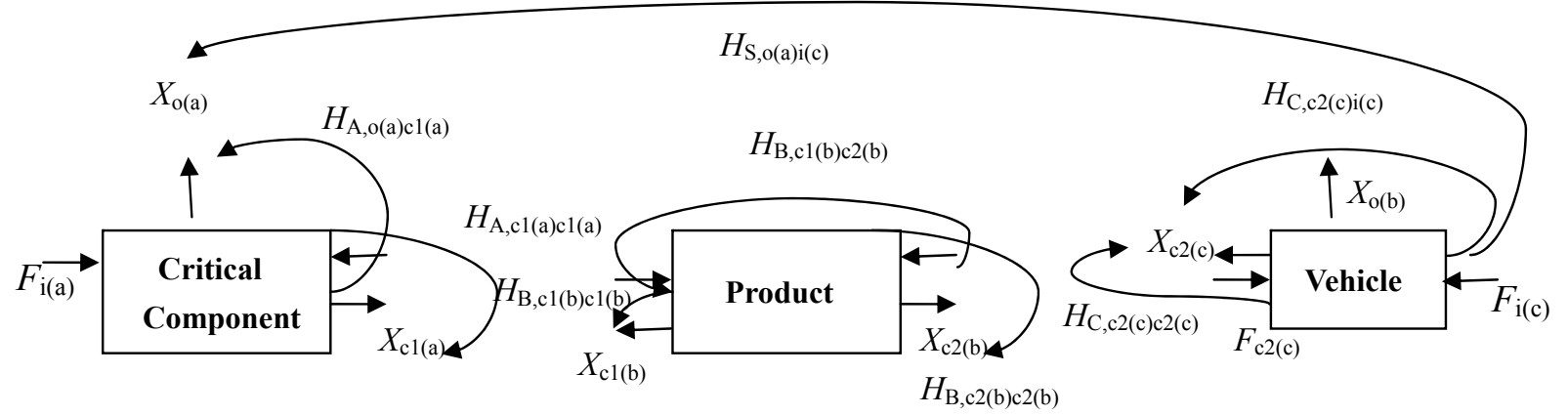

Fig. 9. Signification of the FRFs of the product-transport-system.

The joint part between critical component and main body of product was idealized as a linear spring with stiffness coefficient and damping ratio. To find out the values of them, we developed an inverse substructuring method [16, 17] for packaging system.

For a three-component coupled product-transport-system (A,B,C representing the critical component, the main body of product and the vehicle, respectively) as shown in Fig. 8, the general equations of motion governing the dynamic response of the coupled system is

$$
\left[M_{S}\right]\left\{\ddot{X}_{S}\right\}+\left[C_{S}\right]\left\{\dot{X}_{S}\right\}+\left[K_{S}\right]\left\{X_{S}\right\}=\left\{F_{S}\right\}
$$

And then the system-level response in frequency domain under the action of periodic excitation can be written as

$$
\left\{X_{S}\right\}=\left(-\omega^{2}\left[M_{S}\right]+j \omega\left[C_{S}\right]+\left[K_{S}\right]\right)\left\{F_{S}\right\}=\left[H_{S}\right]\left\{F_{S}\right\}
$$

where $\omega(=2 \pi f)$ is the excitation frequency, and $H_{S}$ is the Frequency Response Function.

By enforcing the force equilibrium of coupling coordinates and applying the traditional inverse substructuring method, we get the prediction formulae for dynamic components of joint part and coupling packaging interface

$$
\begin{gathered}
K_{A B}=\begin{array}{c}
H_{S, c_{1}(a) c_{1}(b)} \\
H_{S, c_{1}(a) c_{1}(a)} H_{S, c_{1}(b) c_{1}(b)}-H_{S, c_{1}(a) c_{1}(b)}^{2} \\
K_{B C}= \\
H_{S, c_{2}(b) c_{2}(c)} \\
H_{S, c_{1}(b) c_{2}(b)} H_{S, c_{2}(c) c_{2}(c)}-H_{S, c_{2}(b) c_{2}(c)}^{2}
\end{array}
\end{gathered}
$$

The signification of the FRFs in the formulae above is depicted in Fig. 9.

In order to verify the veracity of the prediction formula, a physical prototype is manufactured for experimental test, as shown in Fig. 10. The excitation is produced using a modal hammer, while the vibration response is measured using a lightweight accelerometer. The test system is shown in Fig. 11. All of the system-level FRFs needed are 

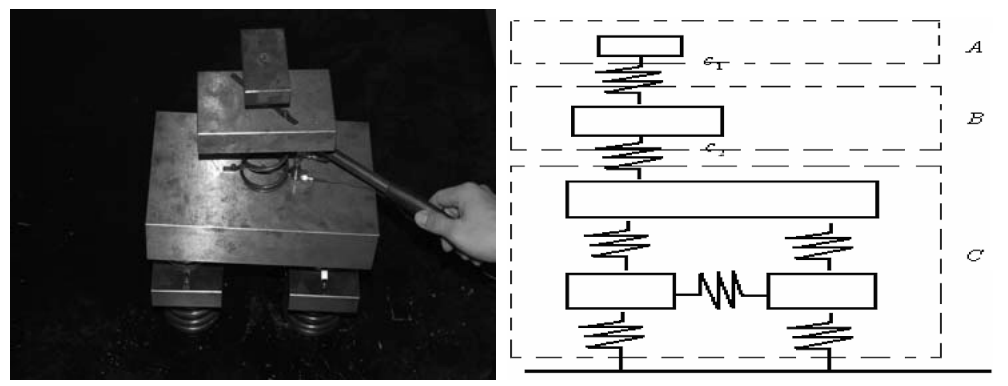

Fig. 10. Photo and model of prototype.

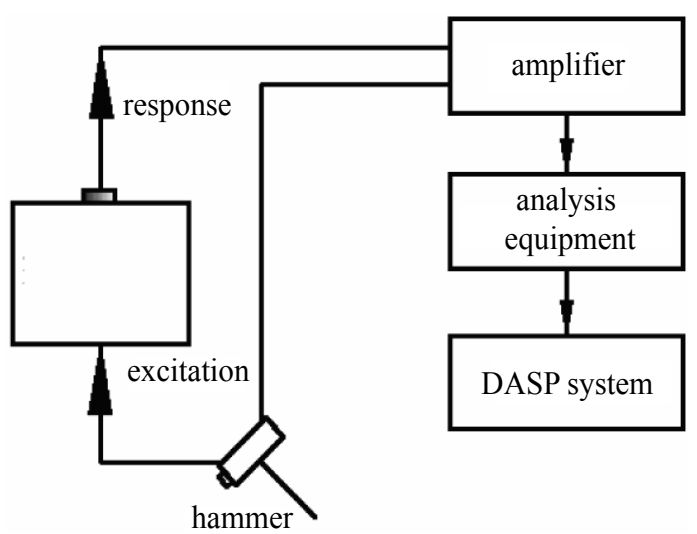

Fig. 11. Test system.

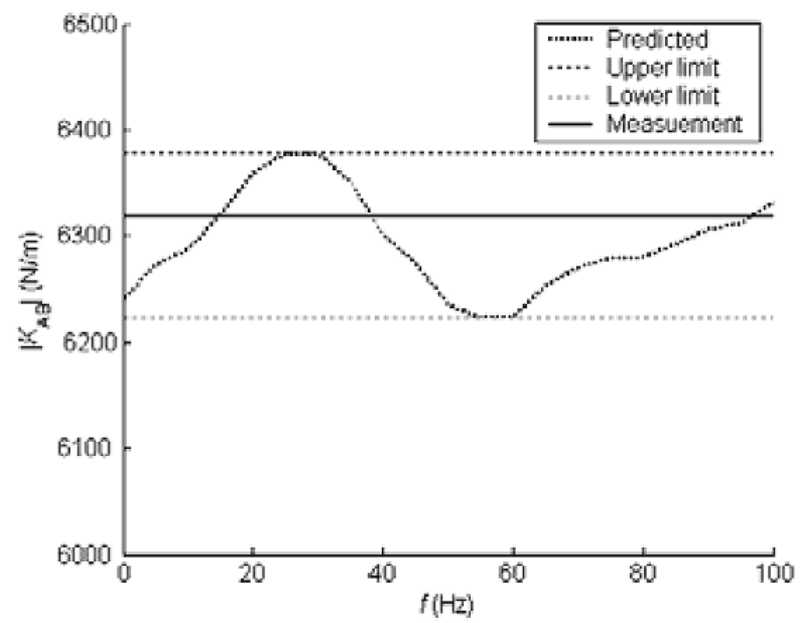

Fig. 12. Comparison of predicted and measured component level FRF (- - - -, predicted; - , measured).

tested. Then the measured system-level FRFs are substituted into Eqs $(11,12)$ to predict the coupling stiffness, and the predicted value is finally compared with that of directly measured, as shown in Fig. 12.

It's shown that the proposed inverse substructuring method can effectively predict the values of the dynamic components. The errors may due to the fact that: (1) the placement of accelerometer cannot be precisely at the interface coupling coordinate; (2) only the vibration response at the Z-direction is measured; (3) the rotational coupling effect is neglected; (4) other unavoidable measuring error. 


\section{Conclusions}

This paper suggested a novel model to represent the packaging system, in which the most fragile part of the product was treated as critical component and all other parts were treated as main body of product.

The research indicated that the shock response of critical component depends much on the defined frequency parameter ratio. Then a concept of three-dimensional shock spectrum was proposed to describe the shock characteristics of critical component, with frequency parameter ratio as a new evaluation parameter. It's found that the shock response of critical component can be effectively reduced by decreasing the defined system parameter $(\beta)$ and/or increasing the damping ratio of cushioning materials. When the frequency parameter ratio is low, the shock response can also be weakened by increasing the damping ratio of critical component.

\section{Acknowledgements}

The authors would like to gratefully acknowledge the support of this research by National Natural Science Foundation of China (Granted No.: 50775100) and Project supported by the Fundamental Research Funds for the Central Universities. The authors would also like to thank for the help of Dr. Fang Duan of Jiangnan University for English writing.

\section{References}

[1] R.E. Newton, Fragility assessment theory and practice[R], Monterey Research Laboratory, Inc. Monterey, California, 1968

[2] G.J. Burgess, Product Fragility and Damage Boundary Theory[J], Packaging Technology and Science 15(10) (1988), 5-10.

[3] D.L. Goodwin, Fatigue damage boundary: an application using trapezoidal shock pulses to generate damage in nonlinear components[C], 21st IAPRI Symposium, Valencia, 2003.

[4] Z.L. Wang, C.F. Wu and D.C. Xi, Damage boundary of a packaging system under rectangular pulse excitation[J], Packaging Technology and Science 11 (1998), 189-202.

[5] Z.W. Wang, On evaluation of product dropping damage[J], Packaging Technology and Science 15 (2002), $115-120$.

[6] L.X. Lu and Z.W. Wang, Dropping bruise fragility and bruise boundary of apple fruit[J], Transaction of ASABE 50(4) (2007), 1323-1329.

[7] M.P. Daum, Application of the shock response spectrum to product fragility testing[D]: [Masters Thesis], Michigan:Michigan State University, 1994.

[8] Z.W. Wang and C.Y. Hu, Shock spectrum and damage boundary curves for nonlinear package cushioning system[J], Packaging Technology and Science 12(5) (1999), 207-217.

[9] M.P. Daum, Combining a fatigue model with a shock response spectrum algorithm[J], Journal of Testing and Evaluation 32(5) (2004), $1-5$.

[10] W.J. Ding, Vibration-absorptive theory[M]. Beijing:Tsinghua University Press, 1988.

[11] A.F. Vakakis and R.H. Rand, Normal modes and global dynamics of a two-degree-of-freedom nonlinear system. Part I: low energies[J], International Journal of Nonlinear Mechanics 27(5) (1992), 861-874.

[12] L. Cveticanin, Vibrations of a coupled two-degree-of-freedom system[J], Journal of Sound and Vibration 247(2) (2001), $279-292$.

[13] L. Cveticanin, The motion of a two-mass system with nonlinear connection[J], Journal of Sound and Vibration 252(2) (2002), 361-369.

[14] L. Cveticanin, Forced nonlinear vibrations of a symmetrical two-mass system[J], Journal of Sound and Vibration 265(2) (2003), 451-458.

[15] J.H. Jiang, Dynamical analysis of dropping shock for packaging system with critical component[D]: [Phd Dissertation]. Wuxi: Jiangnan University, 2009.

[16] Z.W. Wang, J. Wang and C.Y. Hu, Investigation into the dynamical characteristics of packaging system based on critical component by applying the inverse substructuring method [C]. 16th IAPRI Conference, Bangkok, 2008.

[17] J. Wang and Z.W. Wang, Inverse substructure method of three-component coupled product-transport-system[J]. Unpublished, 2009.

[18] S. Goyal and J.M. Papadopoulos, Safe zones for shock-protection of fragile components during impact induced clatter[J], Shock and Vibration 9 (2002), 265-276.

[19] S. Goyal, J.M. Papadopoulos and P.A. Sullivan, Shock protection of portable electronic products: shock response spectrum, damage boundary approach, and beyond[J], Shock and Vibration 4(3) (1997), 169-191. 

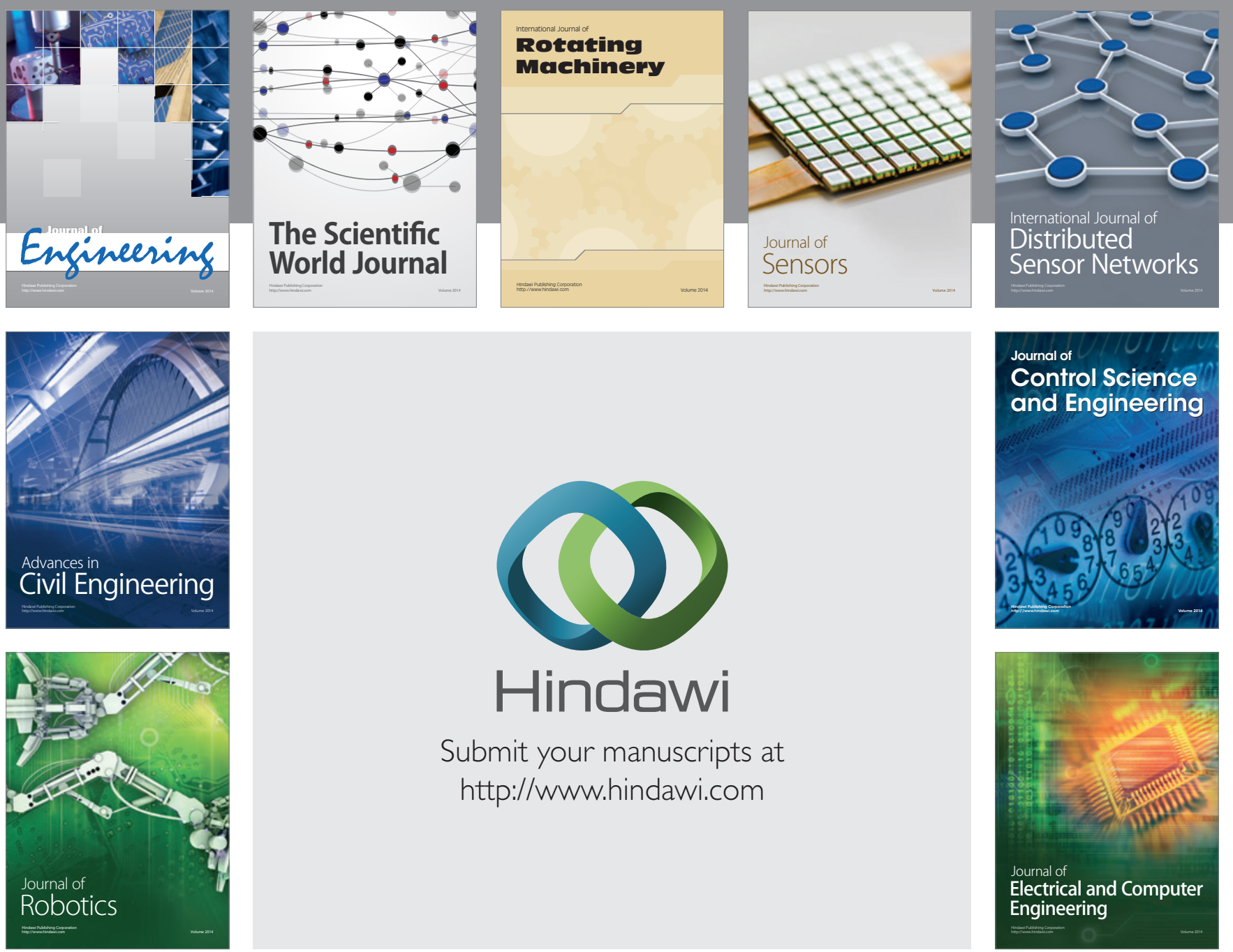

Submit your manuscripts at

http://www.hindawi.com
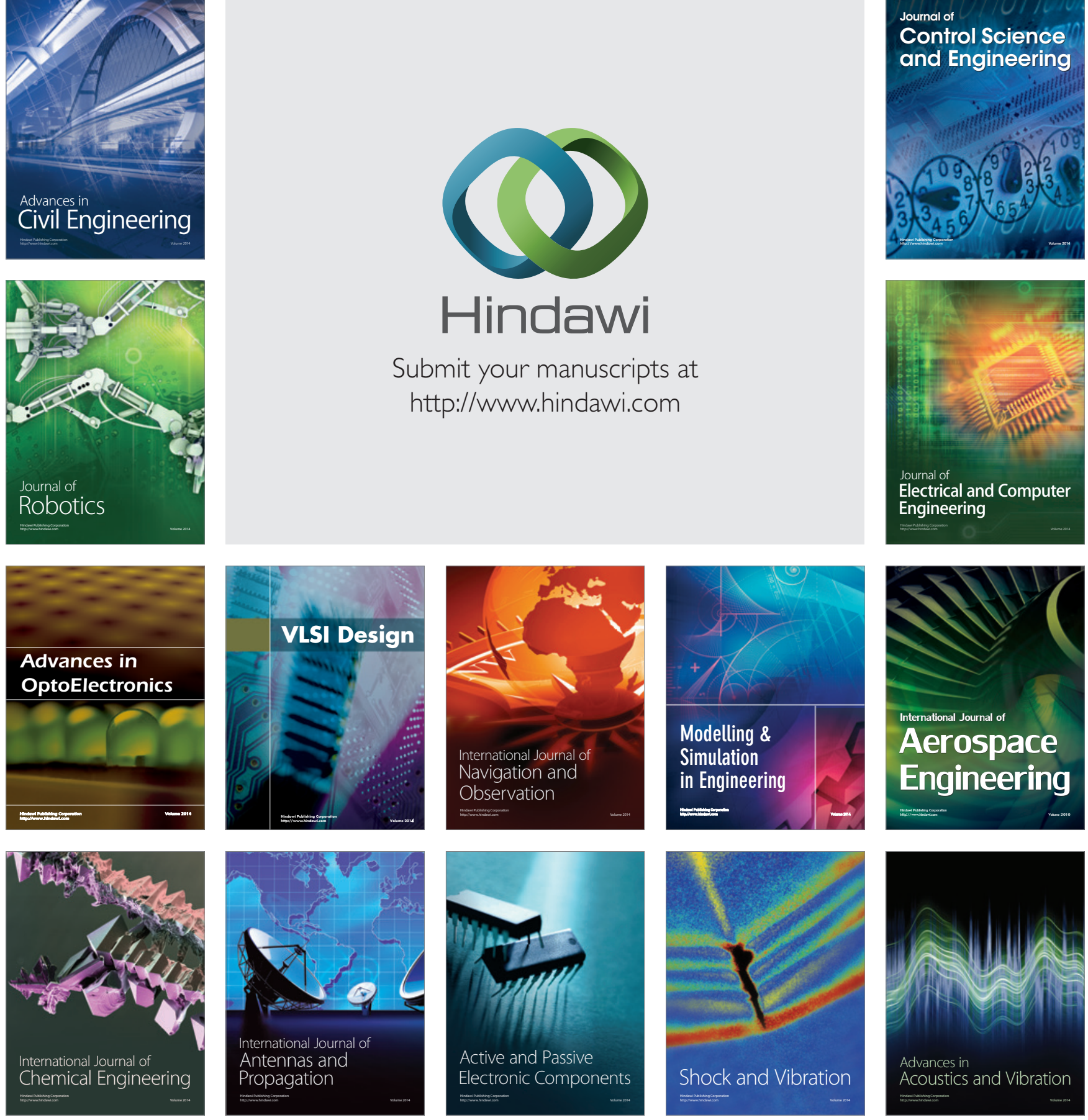